Document downloaded from:

http://hdl.handle.net/10251/137416

This paper must be cited as:

Candelas Valiente, P.; Fuster Escuder, JM.; Pérez-López, S.; Uris Martínez, A.; Rubio Michavila, C. (2019). Observation of ultrasonic Talbot effect in perforated plates. Ultrasonics. 94:281-284. https://doi.org/10.1016/j.ultras.2018.08.019

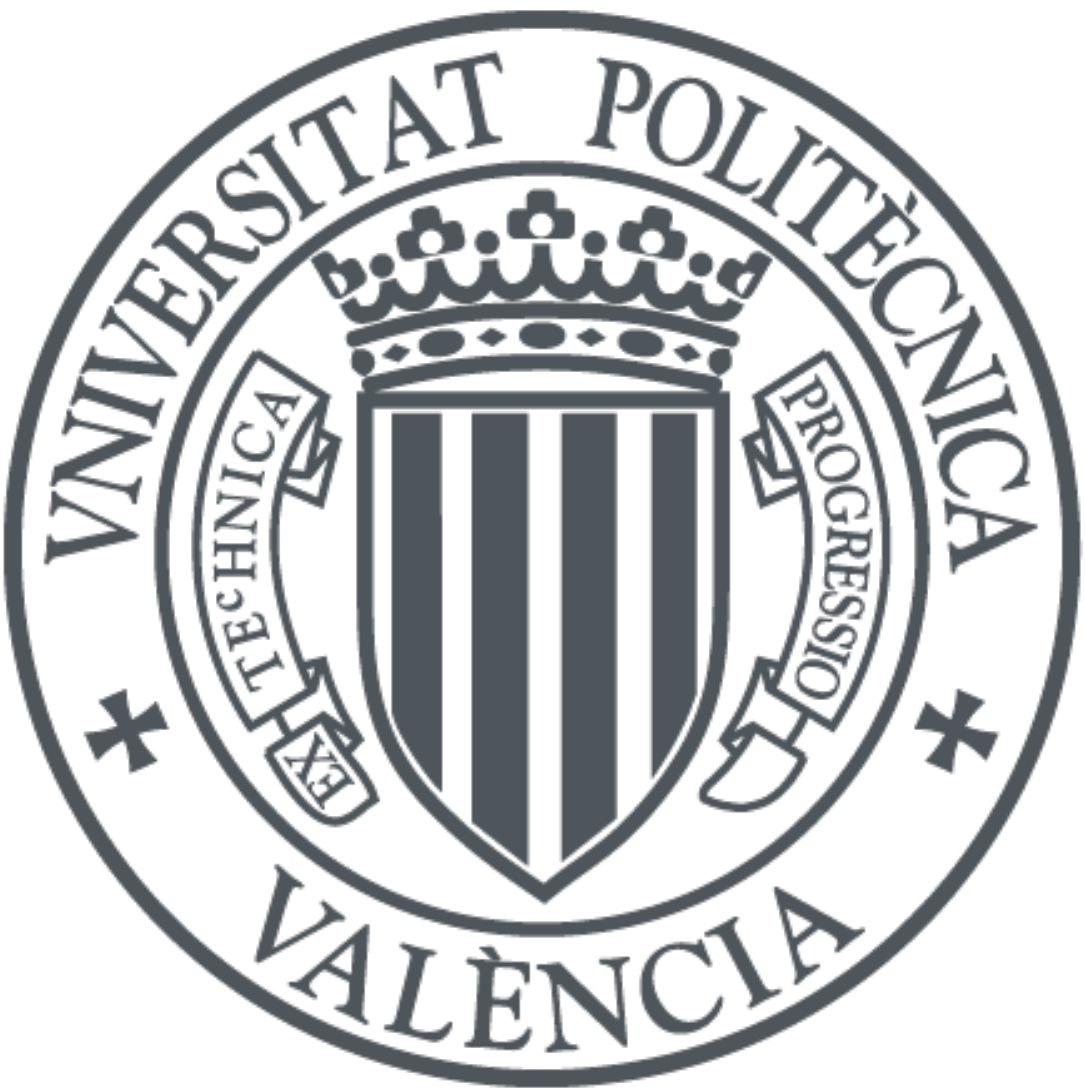

The final publication is available at

https://doi.org/10.1016/j.ultras.2018.08.019

Copyright Elsevier

Additional Information 


\title{
OBSERVATION OF ULTRASONIC TALBOT EFFECT IN PERFORATED
}

\section{PLATES}

Pilar Candelas, José Miguel Fuster, Sergio Pérez-López, Antonio Uris and Constanza Rubio*

Centro de Tecnologías Físicas: Acústica, Materiales y Astrofísica. División Acústica.

Universitat Politécnica de Valencia. Camino de Vera s/n. 46022 Valencia, Spain.

Phone: 34.963877528; Fax: 34.963879525; E-mail: crubiom@fis.upv.es

*Corresponding author.

\begin{abstract}
The Talbot effect using ultrasonic waves transmitted through a periodic perforated plate is presented. The ultrasonic wave-field at the exit of the perforated plate replicates the perforated patttern at the Talbot distance. Experimental results are validated by an analytical model. The measured propagating wave-field is consistent with the analitycal results. The influence of the grating size and the filling fraction on the Talbot effect are also studied, and it is found that both parameters affect the formation of Talbot images.
\end{abstract}

Keywords: Talbot effect, periodic grating, hole array, ultrasonic transmission. 


\section{INTRODUCTION}

The scientific interest in wave interaction with periodic structures began in the XIX century. The pioneering works involved electromagnetic waves. In 1936, Henry Fox Talbot observed that when a monochromatic light plane wave incides on a periodic grating, the transmitted wave-field at the exit of the grating replicates the periodic structure at multiples of a certain distance, namely a self-image of the periodic grating occurs. This phenomenon was named the Talbot effect [1] and the distance at which the self-image is observed, is known as the Talbot length. A few decades ago, in 1881, Lord Rayleigh [2] demonstrated that the Talbot effect was a natural consequence of the Fresnel diffraction mechanism and showed that the Talbot length can be found using $z_{T}=\frac{\lambda}{1-\sqrt{1-\frac{\lambda^{2}}{a^{2}}}}$, where $\lambda$ is the wave length of the incident ligth and $a$ is the grating period. In the paraxial approximation $a \gg \lambda$, the Talbot distance $z_{T}$ is given by $z_{T}=\frac{2 a^{2}}{\lambda}$.

Since the Talbot effect can be observed in both ligth and atoms [3], it has applications in various fields, such as optical lithography [4], scanning electron microscope imaging [5], Bose-Eisntein condensate [6], electron interferometry [7] and X-ray [8]. The plasmon Talbot effect was also predicted [9] and demonstrated with self-imaging experiments [10].

Although research in the fields mentioned above is abundant,there is a lack of studies in the acoustic field. Currently, one of the most interesting fields for the acoustic scientific community is the study of acoustic metamaterials. This type of periodic structures is important because of its technological applications in noise control and its efficiency improvement in the manufacturing of ultrasonic lenses and other devices [11]. That is why the understanding of sound wave diffraction through periodic structures can help the development of new properties and applications of acoustic metamaterials. 
In this work, an observation of the Talbot effect on ultrasonic waves propagating through a periodic structure is reported. A perforated plate with periodically distributed holes is employed. An analytical model is used to validate the experimental results and the influence of the grating size and the filling fraction on the Talbot effect are studied analytically.

\section{MATHEMATICAL FORMULATION OF THE TALBOT EFFECT}

When considering harmonic dependence, the Helmholtz's equation becomes:

$$
\nabla\left(\frac{1}{\rho} \nabla p\right)=\frac{\omega^{2}}{\rho c^{2}} p \quad \Rightarrow \quad\left[\nabla^{2}-\left(\frac{\omega}{c}\right)^{2}\right] p=0
$$

where $\rho$ is the density of the material, $\mathrm{p}$ is the acoustic pressure, $\omega$ is the angular frequency and $\mathrm{c}$ is the speed of sound in the medium

In fact, the grating of a periodic function (in the y direction, see figure 1) can be expressed in terms of a Fourier series as:

$$
p(y, o)=\sum_{n} p_{n} \exp \left[i \frac{2 \pi y_{n}}{a}\right]
$$

being $a$ the grating period.

According to [9], the diffracted plane wave from the grating towards the $z$ direction, can be expressed as:

$$
p(y, z)=\sum_{n} p_{n} \exp \left[i \frac{2 \pi y_{n}}{a}\right] \exp \left[i \frac{2 \pi \xi_{n} z}{\lambda}\right]
$$

where $\xi_{n}=\sqrt{1-\left(\frac{n \lambda}{a}\right)^{2}}$ and $\lambda$ is the wavelength

When analysing Fresnel diffraction, the binomial expansion

$$
\frac{\xi_{n}}{\lambda}=\frac{1}{\lambda}-\frac{n^{2}}{z_{T}}-\left(\frac{\lambda}{p}\right)^{2} \frac{n^{4}}{4 z_{T}}-\cdots
$$

can be truncated at the term proportional to $n^{2}$. Then, equation 1 can be rewritten as

$$
p(y, z)=\exp \left[i \frac{2 \pi z}{\lambda}\right] \sum_{n} p_{n} \exp \left[i \frac{2 \pi y_{n}}{p}\right] \exp \left[-i \frac{2 \pi n^{2} z}{\lambda}\right]
$$


From the latter expression, it can be deduced that

$$
\begin{gathered}
p\left(y, z_{T}\right)=\exp \left[i \frac{2 \pi z}{\lambda}\right] p(y, 0) \\
p\left(y, \frac{z_{T}}{2}\right)=\exp \left[i \frac{2 \pi z}{\lambda}\right] p\left(y-\frac{z_{T}}{2}, 0\right)
\end{gathered}
$$

\section{EXPERIMENTAL SETUP}

The basic periodic structure was built using a $350 \times 450 \mathrm{~mm}^{2}$ brass plate drilled with circular holes of diameter $d=3 \mathrm{~mm}$, periodically distributed in a square array with period $a=6 \mathrm{~mm}$, being the filling fraction $f f=0,196$. The filling fraction for a squared array is given by $f f=\frac{\pi d^{2}}{4 a^{2}}$. The thickness of the plate is $2 \mathrm{~mm}$.

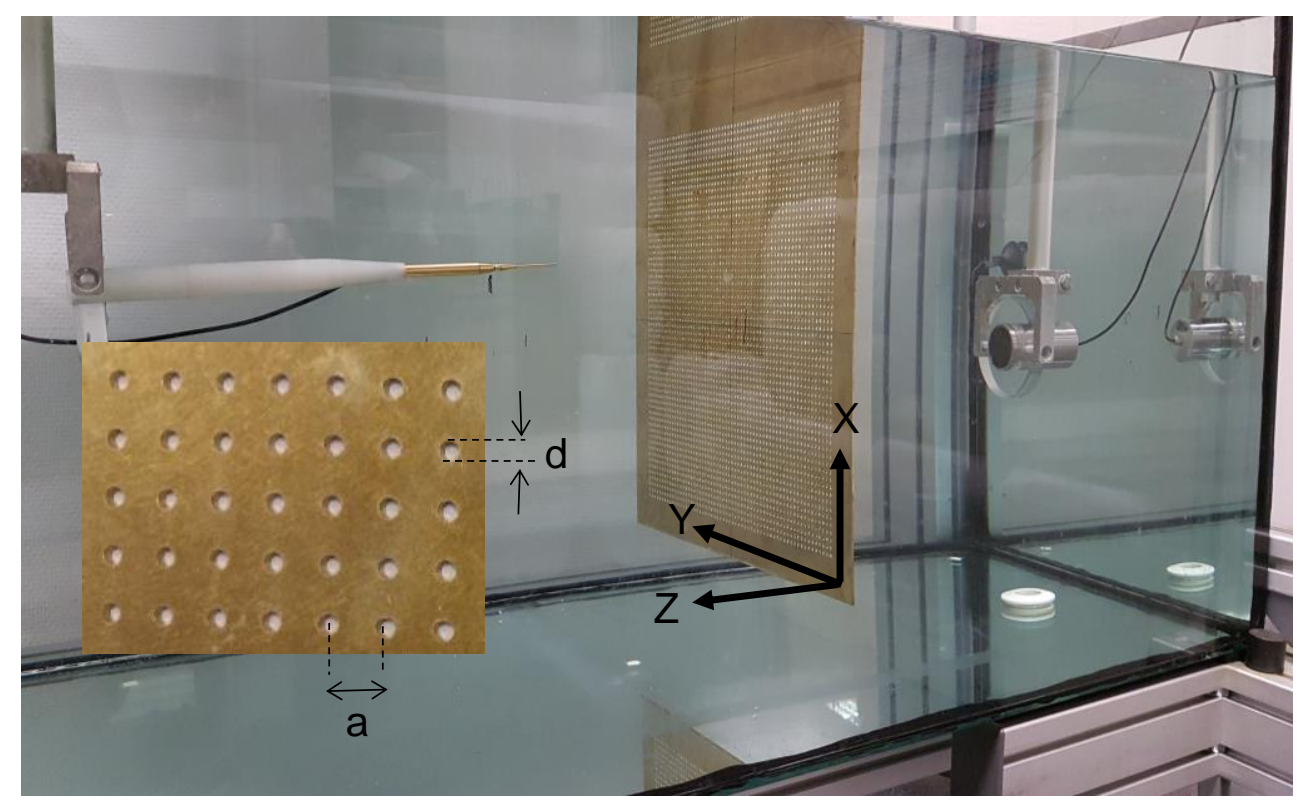

Figure 1. Picture of the experimental setup. The insed shows a picture of the perforated plate.

The measurements were carried out using the ultrasonic immersion transmission technique. The perforated plate was placed in a water tank. To align the sample, an automated positioning system was used. This system which is built around the water tank, aligns and places the hydrophone through a 3D grid of measurement points located at any trajectory inside the tank. A piston transducer (Imasonic) 
centered at a frequency of $250 \mathrm{kHz}$, with an active diameter of $32 \mathrm{~mm}$ was employed as the emitter and a Polyvinylidene fluoride (PVDF) needle hydrophone (Precision Acoustics Ltd.) model HPM1/1 with a diameter of $1.5 \mathrm{~mm}$ and a $\pm 4 \mathrm{~dB}$ bandwidth, spanning from $200 \mathrm{kHz}$ to $15 \mathrm{MHz}$, was used as the receiver. The pulse launched by the emitter piston transducer through the perforated plate was detected by the hydrophone and then, it was acquired by the pulser/receiver, post amplified and digitized by a Picoscope oscilloscope (model 3224). Time domain data was analysed after averaging 100 different measures. A time window was set to be wide enough to collect only direct transmission samples through the perforated plate and avoid indirect transmission measures due to lateral reflections. Scanning was done using the automated positioning system along a plane normal to the plate (XY plane), with a spatial resolution of $0.5 \times 0.5 \mathrm{~mm}^{2}$. Figure 1 shows an image of the experimental setup.

\section{RESULTS}

In order to validate the analytical model presented in Section 2, the brass plate described in the experimental section was used. The square arrangement characteristics of the perforated plate were period $a=6 \mathrm{~mm}$, hole diameter $d=3 \mathrm{~mm}$, and filling fraction $f f=0.196$. An incident plane wavelength $\lambda_{0}=6 \mathrm{~mm}$, that corresponds to the $\lambda_{0}=a$ case, has been analized both analytically (figure 2a) and experimentally (figure $2 \mathrm{~b}$ ). The analytical results show good agreement with the experimental measures. The wave transmission pattern reproduces the self-image corresponding to the Talbot effect. The Talbot length, $z_{T}=\frac{\lambda}{1-\sqrt{1-\frac{\lambda^{2}}{a^{2}}}}$, is $6 \mathrm{~mm}$ in this case, which corresponds to a grating period $a=6$ $\mathrm{mm}$ and an incidence wavelength $\lambda_{0}=6 \mathrm{~mm}$. At integer multiples of the Talbot distance, the grating's self-image can be identified. The good agreement between experimental and analytical results enable the experimental validation of the analytical model. 

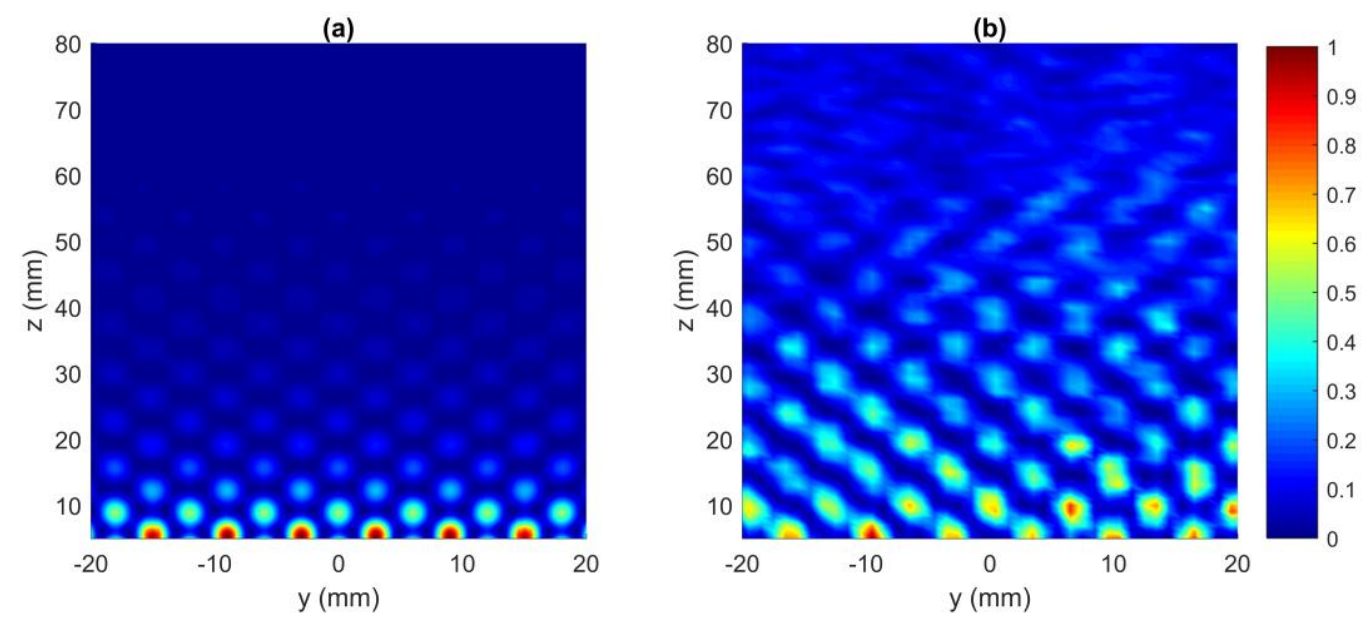

Figure 2. (a) Analytical results Experimental carpet of Talbot self-imaging (b) Experimental results

Once the analytical model has been validated, the influence of the grating size and the filling fraction on the Talbot effect is investigated. First, the grating size is considered. In order to analyze the influence of the grating size on the Talbot effect, the diffration intensity of two grating sizes, $\mathrm{L}_{1}=350 \mathrm{~mm}$ and $\mathrm{L}_{2}=$ $3500 \mathrm{~mm}$, at their Talbot distances are studied. Figure 3 shows the simulated results reproducing the self-imaging pattern, where the wavelength of the incident wave is $6 \mathrm{~mm}$, the grating period is $6 \mathrm{~mm}$ and the hole diameter is $3 \mathrm{~mm}$. Figure 4 shows the transverse diffraction intensities corresponding to the Talbot effect at different Talbot lengths, $\mathrm{z}_{\mathrm{T}}$. It can be observed that there are differences in the diffraction intensity distribution for both grating sizes. However, as the propagation distances increases, diffraction intensities vary with the grating size. As the grating size becomes smaller, there is a reduction in the diffraction intensity, which becomes more significant as the distances increase. The intensity at a certain distance is due to the contributions of many holes on the plane within an area. As the distance increases, the grating size influence is more evident as the area becomes larger. Therefore, the influence of the grating size on the diffraction intensity is very significant when analyzing the Talbot effect. 

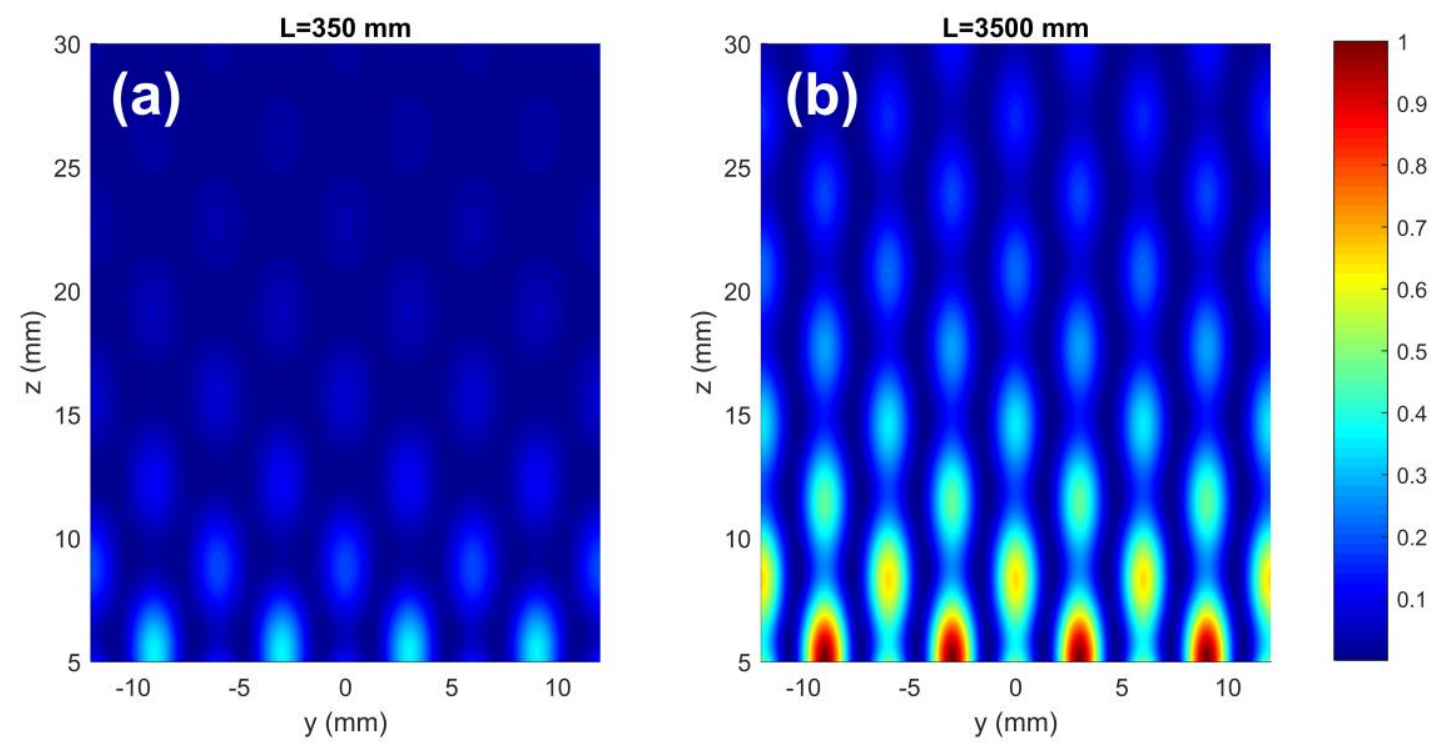

Figure 3. Carpet of Talbot self-imaging for grating size (a) $\mathrm{L}_{1}=350 \mathrm{~mm}$ and (b) $\mathrm{L}_{2}=3500 \mathrm{~mm}$
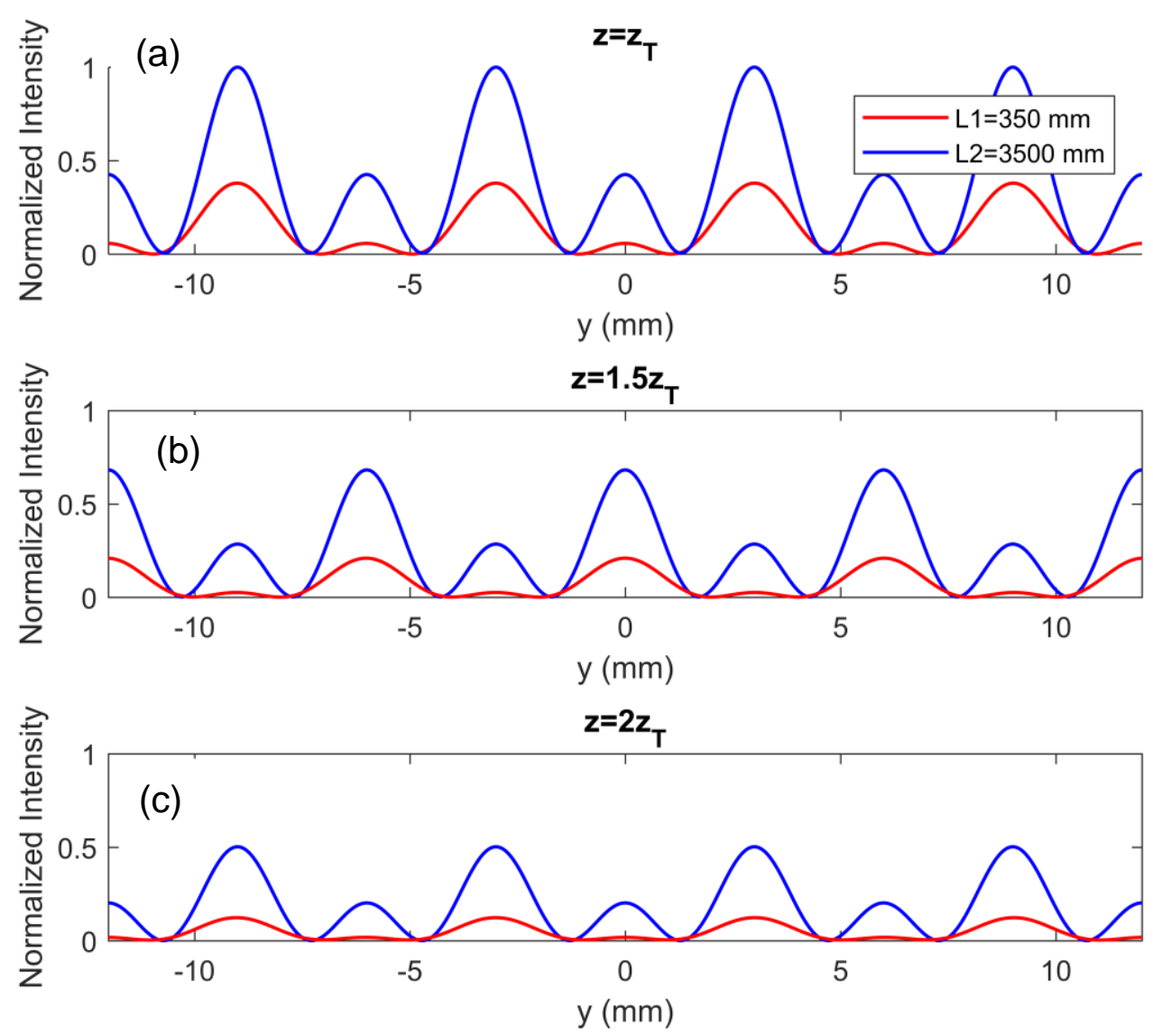

Figure 4. Comparison of transverse diffraction intensity distribution for both grating size $\mathrm{L}_{1}=350 \mathrm{~mm}$ and $\mathrm{L}_{2}=3500 \mathrm{~mm}$ at different Talbot distances, $\mathrm{zT}_{\mathrm{T}}$, (a) $\mathrm{z}=\mathrm{ZT}_{\mathrm{T}}$, (b) $\mathrm{z}=1.5 \mathrm{ZT}_{\mathrm{T}}$ and (c) $\mathrm{z}=2 \mathrm{ZT}_{\mathrm{T}}$. 
To analyze the influence of the filling fraction on the formation of the Talbot images, calculations have been carried out keeping the grating period fixed and varying the holes diameter. A grating period of 9 $\mathrm{mm}$ and four different diameters have been chosen: $2 \mathrm{~mm}, 3 \mathrm{~mm}, 4.5 \mathrm{~mm}$ and $5 \mathrm{~mm}$, being the filling factors $0.0387,0.0872,0.1963$ and 0.2424 , respectively. Figure 5a shows the results of the transverse diffraction intensity distribution at the Talbot distance $\left(\mathrm{z}=\mathrm{z}_{\mathrm{T}}\right)$ at a frequency of $250 \mathrm{kHz}$. As it can be observed, when the filling fraction augments, the diffraction intensity increases as expected. Likewise, a widening of the intensity peaks is also observed as the filling factor increases. However, when the frequency is increased up to $500 \mathrm{kHz}$, as shown in Figure 5b, it is observed that an undulation takes place at the intensity peaks, which decreases as the filling fraction increases. This phenomenon is due to the diffraction orders. As the filling fraction increases, due to an increase in the hole diameter, a greater number of diffraction orders meet again at $\mathrm{z}=$ zт. However, when the hole diameter is reduced, the higher diffraction orders are truncated, resulting in the undulation phenomenon.
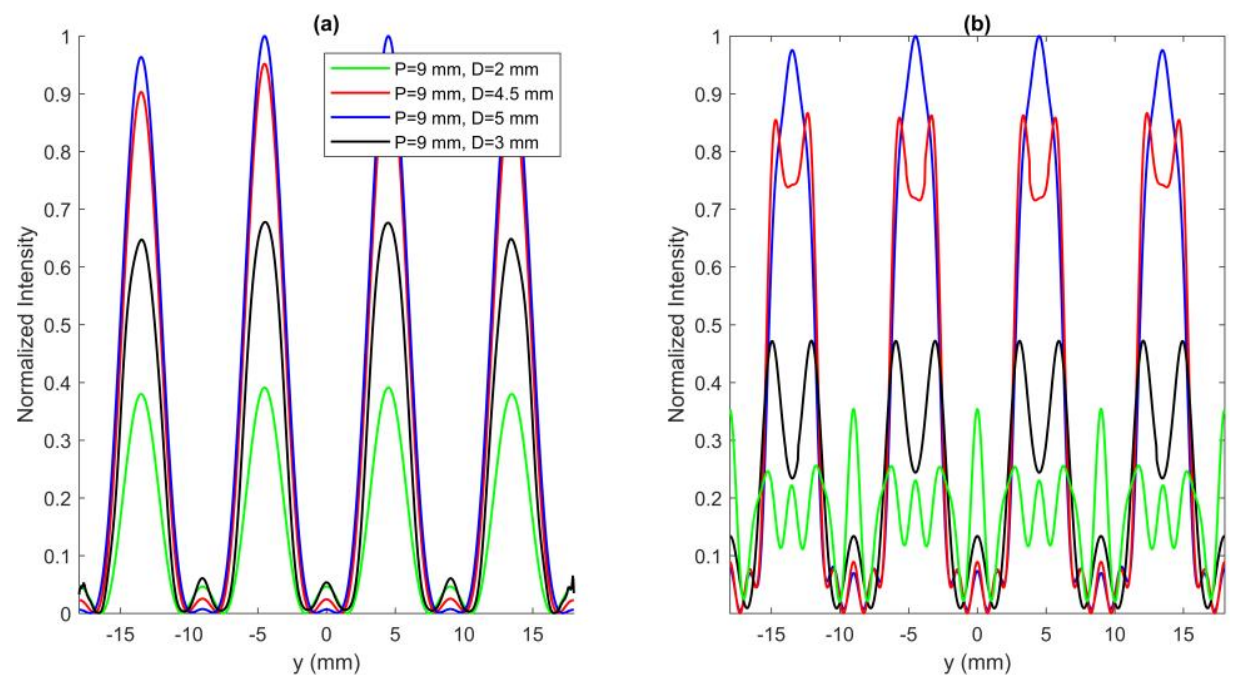

Figure 5. Transverse diffraction intensity distribution at $\mathrm{z}=\mathrm{z}_{\mathrm{T}}$ for (a) $250 \mathrm{kHz}$ and (b) $500 \mathrm{kHz}$

\section{5.- CONCLUSIONS}


In conclusion, the influence of the grating size and the filling fraction on the Talbot effect has been analytically studied. To obtain ideal Talbot images of the grating, the size of the grating must be large enough, since intensity comes from the contributions of many holes on the plane within a certain area. The filling fraction is another parameter that influences the formation of Talbot images. It has been found that, as it could be expected, the intensity at the Talbot distances increases as the filling fraction augments. When frequency increases, and the filling fraction is reduced, the higher diffraction orders are truncated, resulting in an undulation phenomenon in the diffraction intensity distributions.

\section{Acknowledgements}

This work was financially supported by the Spanish MINECO through project TEC2015-70939R.

\section{REFERENCES}

[1] F.F. Talbot, Facts Relating to Optical Science, Philos. Mag. 9, (1836), 401-407.

[2] L. Rayleigh, On Copying Diffraction Gratings and some Phenomena Connected Therewith, Philos. Mag. 11, (1881), 196-205.

[3] M. S. Chapman, C.R. Ekstron, T.D. Hammond, J. Schmiedmayer, B.E. Tannian, S. Wehinger, D.E. Pritchard, Near field imaging of atom diffraction gratins: The atomic Talbot effect, Phys. Rev. A, 51(1), (1995), R14-R17.

[4] L. Stuerzebecher, T. Harzendorf, U. Vogler, U.D. Zeither, R. Voelker, Advanced mask aligner lithography fabrication of periodic patterns using pinhole array mask and Talbot effect, Opt. Express 18(19), (2010), 19485-19494.

[5] H. Kim, W.Li, S. Danylyuk, W.S. Brocklesby, M.C. Marconi, L. Juschkin, Optical properties of 2D fractional Talbot patterns under coherent EUV illumination, J. Phys. D. Appl. Phys. 48, (2015), 375101. 
[6] L. Deng, E.W. Hagley, J. Denschlag, J.E. Simsarian, M. Edwards, C.W. Clark, K. Helmerson, S.L. Rolston, W.D. Phillips, Temporal, matter-wave-dispersion Talbot effect, Phys. Rev.Lett. 83, (1999), $5047-5411$.

[7] B. J. McMorran, A. D. Cronin, An electron Talbot interferometer, N. J. Phys. 11, (2009), 033021.

[8] F. Pfeiffer, T. Weitkamp, O. Bunk, C. David, Phase retrieval and differential phase-contrast imaging with low brilliance X-ray sources, Nature Phys. 2, (2006), 258-261.

[9] M. R. Dennis, N. I. Zheludev, F. Javier Garcia de Abajo, The plasmon Talbot effect”, Opt. Express 15, (2007), 9692-9700.

[10] W. Zhang, C. Zhao, J. Wang, J. Zhang, An experimental study of the plasmonic Talbot effect, Opt. Express 17, (2009), 19757-19762.

[11] J.L. Aragon, R. Quintero-Torres, J.L. Domínguez-Juárez, E. Iglesias, S. Ronda, F. Montero de Espinosa, Planar modes free piezoelectric resonators using a phononic cristal, Ultrasonics 71, (2016), 177-182. 


\section{FIGURES CAPTIONS}

Figure 1. Picture of the experimental setup. The insed shows a picture of the perforated plate.

Figure 2. (a) Analytical results Experimental carpet of Talbot self-imaging (b) Experimental results

Figure 3. Carpet of Talbot self-imaging for grating size (a) L1 $=350 \mathrm{~mm}$ and (b) L2 $=3500 \mathrm{~mm}$

Figure 4. Comparison of transverse diffraction intensity distribution for both grating size $\mathrm{L}_{1}=350 \mathrm{~mm}$ and $\mathrm{L}_{2}=3500 \mathrm{~mm}$ at different Talbot distances, $\mathrm{z}_{\mathrm{T}}$, (a) $\mathrm{z}=\mathrm{z}_{\mathrm{T}}$, (b) $\mathrm{z}=1.5 \mathrm{z}_{\mathrm{T}}$ and (c) $\mathrm{z}=2 \mathrm{z}_{\mathrm{T}}$.

Figure 5. Transverse diffraction intensity distribution at $\mathrm{z}=\mathrm{z}_{\mathrm{T}}$ for (a) $250 \mathrm{kHz}$ and (b) $500 \mathrm{kHz}$ 